\title{
Management in fünf Dimensionen
}

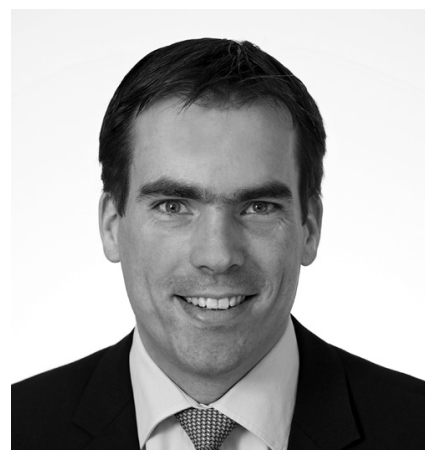

VON SVEN LÜNGEN

Sven Lüngen ist Sozialpädagoge und Betriebswirt. Er ist Geschäftsführer des Alexander-Stifts, einem großen Altenhilfeträger in BadenWürttemberg, Tochtergesellschaft der Diakonie Stetten. www.alexander-stift.de

\author{
Das Management in der Sozialwirtschaft sieht sich \\ meistens mehrfachen Anforderungen gegenüber, \\ die sich in der Regel nicht problemlos miteinander \\ verbinden lassen. Abwägen, Bewerten und ethische \\ Entscheidungen treffen, welcher Dimension in welcher \\ Situation der Vorrang einzuräumen ist, wird somit \\ eine zentrale Voraussetzung für Führungskräfte. \\ Fünf Aspekte können dabei hilfreich sein.
}

Aus dem Alltag der Sozialwirtschaft: Ein Jugendlicher ist unglücklich in seiner ambulant betreuten Wohnung und fühlt sich von den pädagogischen Mitarbeitern ungerecht behandelt. Das Gebäude eines Krankenhauses ist alt und über das Leck in dem maroden Heizungstank gelangt unbemerkt Heizöl ins Grundwasser. Die von Pflegesatzverhandlung zu Pflegesatzverhandlung sich verschlechternde Refinanzierungssituation eines Altenpflegeheimes verleiten den Geschäftsführer dazu, über eine Tochtergesellschaft den bestehenden Tarifvertrag zu umgehen, um Personalkosten zu reduzieren. Stress, Druck und Angst prägen den Arbeitsalltag einer Pflegehilfskraft. »Es der Chefin recht zu machen « erscheint ihr wichtiger, als sich persönlich weiterzuentwickeln. Eine Heimleiterin setzt sich jeden Tag für die Menschen mit Behinderung ihres Hauses ein, unterstützt die Mitarbeitenden wo sie nur kann und ist für Angehörige immer erreichbar - nun befindet sie sich in stationärer Behandlung einer Spezialklinik für Burn-out.

In allen diesen Situationen stellt sich die Frage, wer für was die Verantwortung trägt und wie Führungskräfte sozialer Einrichtungen den unterschiedlichsten Anforderungen zahlreicher Interessensgruppen in komplexen Situationen gerecht werden können. In der
Unterschiedlichkeit der Beispiele wird deutlich, dass das Management sozialer Einrichtungen mehreren anspruchsvollen Verantwortungsdimensionen gerecht werden muss: Lebensqualität der Menschen fördern, der Umwelt und dem Umfeld nicht schaden, Lebensfähigkeit der Organisation absichern, Wachstum der Mitarbeitenden, eigene Leistungsfähigkeit und Gesundheit fördern.

Es sind mehrfache Anforderungen, die sich in der Regel nicht friktionslos miteinander verbinden lassen. Abwägen, Bewerten und ethische Entscheidungen treffen, welcher Dimension in welcher Situation der Vorrang einzuräumen ist, wird somit eine zentrale Voraussetzung für ein verantwortungsvolles Management in Sozialunternehmen sein.

Ein verkürzter Blick auf nur eine Dimension führt schnell zu einer unzureichenden und ungerechten Problemwahrnehmung. Zumal dabei die Gefahr besteht, dass immer die Dimension in den Fokus von Führungskräften gerät, die aktuell am vehementesten von engagierten Interessensgruppen vertreten wird. Somit suggerieren auf solch vereinfachten Diagnosen aufbauende Bemühungen zwar vordergründig Handlungs- und Lösungskompetenz des Managements, doch die Ausgangslage ist oft komplexer und multipolar. Nachfolgend werden die genannten fünf Dimensionen skizziert und zur Diskussion gestellt. Sie sollen 
Ansätze für die Umsetzung eines verantwortungsvollen Managements markieren.

\section{Erste Dimension verantwortungsvollen Managements: Lebensqualität der Menschen fördern}

Soziale Einrichtungen und Organisationen die soziale Dienstleistungen für Menschen mit Hilfe-, Unterstützungsoder Pflegebedarf erbringen haben ein zentrales Sachziel zu verfolgen: Lebensqualität der Menschen fördern.

Nicht die Erfüllung von Anforderungen oder Vorgaben Dritter oder gar wirtschaftliche Formalziele sollten im Mittelpunkt der Bemühungen stehen, sondern die Menschen - in Zeiten einer immer weiter um sich greifenden Bürokratie und unter dem Deckmantel von »Gerechtigkeit « und »Genauigkeit « von Verrechnungen auch innerhalb von Organisationen wachsenden, quasi selbst verschuldet, unnötige Transaktionskosten keine einfache Aufgabe.

Führungskräfte haben die Verantwortung den Ressourceneinsatz und die Aufmerksamkeit immer wieder auf die Menschen auszurichten. Dort wo etwas für das Wohlbefinden und die Zufriedenheit von Menschen getan werden kann, sollte vereinfacht gesprochen »investiert « werden und dort, wo Initiativen nicht $\mathrm{zu}$ einer verbesserten Lebensqualität beitragen, ist der Ressourceneinsatz $\mathrm{zu}$ hinterfragen und $\mathrm{zu}$ minimieren.

Hierbei ist zu berücksichtigen, dass Wohlbefinden, Zufriedenheit und das Konzept der Lebensqualität rein subjektiv zu bewerten sind. Wir können niemandem anordnen mit einer Leistung deshalb zufrieden zu sein, weil die Mehrheit der Leistungsempfänger mit der erbrachten Qualität einverstanden ist. Persönliche Erwartungen und Einstellungen im Verhältnis zur subjektiven Wahrnehmung der Leistung werden hier entscheidend sein.

Lebensqualität kann somit nur dann wirksam als Orientierung für die Bemühungen in einer sozialen Einrichtung genutzt werden, wenn die Bereitschaft besteht die Produkt- und Dienstleistungsgestaltung möglichst individuell an den persönlichen Bedarfen der Menschen auszurichten und hierzu mit Ihnen in einen offenen Dialog treten.

\section{Zweite Dimension verantwortungsvollen Managements: Der Umwelt und dem Umfeld nicht schaden}

In der Industrie haben sich in den letzten Dekaden Initiativen und Berichtswesen zu einer Corporate Social Responsibility etabliert. In vielen Fällen kann man sich zwar dem Eindruck nicht entziehen, dass hierbei primär Marketing-Überlegungen im Zentrum stehen, doch es finden sich immer wieder Unternehmensbeispiele, die einer umfassenden gesellschaftlichen (ökologischen, sozialen und ökonomischen) Verantwortung gerecht werden wollen. Unter anderen plädiert auch der Managementvordenker Peter Drucker für eine Verpflichtung der Unternehmen für eine verantwortungsvolle Gestaltung der Unternehmens-Umfeld-Beziehungen: »Ein Unternehmen lebt in der Gesellschaft und in der Gemeinschaft, es muss daher soziale Verantwortung zeigen, wenigstens insoweit, als es die Verantwortung für sein Einwirken auf die Umwelt übernimmt.«(1)

Soziale Unternehmen leisten im Kern ihres Auftrages per se einen Beitrag für die Gesellschaft und übernehmen somit auch soziale Verantwortung für Bedürftige verschiedenster Couleur. Doch wie steht es um die ökologischen Auswirkungen der unternehmerischen Tätigkeit? Oder die sozialen Folgen, die beispielsweise für die Mitarbeitenden aus den gewählten Arbeitsbedingungen resultieren?

Bis heute widmen sich die Führungskräfte zahlreicher Sozialunternehmen recht wenig den Überlegungen einer nachhaltigen Betriebsführung. Strategi- sche und operative Fragen der Beurteilung und Entscheidungsfindung bleiben zumeist auf die Aspekte innerhalb der eigenen Organisation begrenzt. Notwendig wäre stattdessen, Themen der sozialen und ökologischen Nachhaltigkeit mit in ein integrales Führungsverständnis aufzunehmen. (2)

Auch nicht beabsichtigte soziale und ökologische Konsequenzen des Engagements müssen die Führungskräfte sozialer Einrichtungen mitverantworten.

\section{Dritte Dimension verantwortungsvollen Managements: Lebensfähigkeit der Organisation absichern}

»Die erste Aufgabe des Managers von morgen wird es sein, Verantwortung für die Leistungsfähigkeit der Institution zu übernehmen, für die er arbeitet.« (3)

Eine Leistungsfähigkeit, die hier und heute und auch in Zukunft in Sozialunternehmen gesichert sein muss, damit auch in den nächsten Jahrzehnten soziale Dienstleistungen für Bedürftige in unserer Gesellschaft erbracht werden können. Eine Leistungsfähigkeit, die somit auch eine langfristige »Lebensfähigkeit « von sozialen Einrichtungen absichern wird.

Führungskräfte müssen das heutige Geschäft sowie die heutigen Konzepte optimieren (Qualität der Leistungen, Marktstellung, Produktivität, aktuelle Verpflichtungen) und möglichst ressourcensparend wirtschaften. Parallel sind jedoch auch Konzepte für die Zukunft zu entwickeln und das Unternehmen auf veränderte Rahmenbedingungen (Inno-

\section{Fünf Thesen für das Management}

1. Verantwortung übernehmen heißt nicht alles zu tun, was für die Menschen in sozialen Einrichtungen wünschenswert ist oder was von Interessensgruppen erwartet wird. Es bedeutet jedoch, sich in Abwägung aller Dimensionen für das Bestmöglichste zu engagieren.

2. Das Management hat nicht die Verantwortung für das oft schwierige Umfeld sozialer Einrichtungen, jedoch dafür, der Umwelt und dem Umfeld möglichst nicht zu schaden und die Rahmenbedingungen der Einrichtung (auch politisch) zum Positiven zu beeinflussen.

3. Verantwortung für den Fortbestand sozialer Einrichtungen zu übernehmen bedeutet auch harte Entscheidungen treffen und durchhalten zu müssen. Den Sinn und die Notwendigkeit dieser vermitteln zu können, wird die Voraussetzung sein, dass Menschen gegebenenfalls notwendige harte Einschnitte mittragen und akzeptieren.

4. Verantwortung für das Wachstum von Mitarbeitenden heißt nicht primär jene zu »entwickeln«, sondern Menschen in ihrem fachlichen und persönlichen Wachstum zu fördern.

5. Eigenverantwortung zu übernehmen setzt den kritischen Blick auf das eigene Handeln und die eigene Person voraus. Nur so kann eine Führungskraft sich auch selbst gerecht werden.

Sven Lüngen 

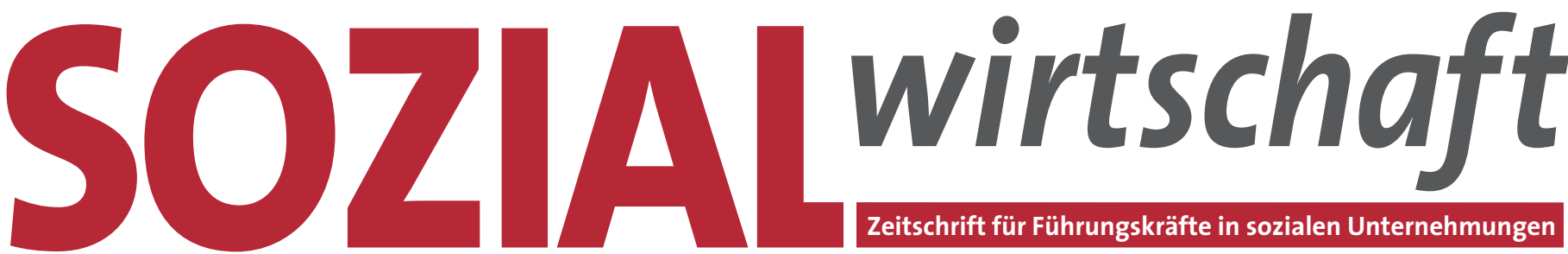

Zeitschrift für Führungskräfte in sozialen Unternehmungen

Herausgegeben von der Bundesarbeitsgemeinschaft der Freien Wohlfahrtspflege e. V.

\section{Jahresinhaltsverzeichnis 2013}

\section{EDITORIAL}

Steuern auf Sicht

SOZIALwirtschaft $1 / 2013$, Seite 5

Mittler zwischen Staat, Markt und Zivilgesellschaft

SOZIALwirtschaft 2/2013, Seite 5

Attraktive Branche

SOZIALwirtschaft 3/2013, Seite 5

\section{Geld im System}

SOZIALwirtschaft 4/2013, Seite 5

Wirkungen und Nebenwirkungen

SOZIALwirtschaft 5/2013, Seite 5

Altes und Neues

SOZIALwirtschaft 6/2013, Seite 5

\section{TITEL}

\section{Managementfehler}

Controlling hilft!

Von Martin Kelter

SOZIALwirtschaft 1/2013, Seite 7-9

\section{Verwaltung}

Schlank und effizient

Von Sandra Winter und Sascha Gründel

SOZIALwirtschaft 1/2013, Seite 10-12

\section{Haftung}

Für den Fall des Falles

Von Benjamin Liedy

SOZIALwirtschaft 1/2013, Seite 13-15

\section{Steuerung}

Leistungserbringung braucht öffentliche Sozialplanung

Von Andreas Strunk

SOZIALwirtschaft 2/2013, Seite 7-9

\section{Ressourcen}

Soziale Dienste brauchen sechs Netzwerke

Von Brigitte Reiser

SOZIALwirtschaft 2/2013, Seite 10-12

\section{Kooperationen}

Fünf Engpässe behindern die Zusammenarbeit

Von Jochen Walter und Günter Raß

SOZIALwirtschaft 2/2013, Seite 13-14

\section{Kooperation}

Von der Anstalt über die Gemeinde zur Integrierten Versorgung Von Klaus-D. Liedke

SOZIALwirtschaft 2/2013, Seite 15-17

\section{Arbeitsmarkt}

Unter dem Spardiktat

Von Tina Hofmann

SOZIALwirtschaft 2/2013, Seite 18-20

\section{Sociosclerose}

Zukunftsfähigkeit gefährdet

Von Josef Hilbert, Michaela Evans und Viacheslav Galchenko

SOZIALwirtschaft 3/2013, Seite 7-9

\section{Personalentwicklung}

Vom Umgang mit Menschen

Von Wolfgang Hoffmann

SOZIALwirtschaft 3/2013, Seite 10-13

Personalmangel

Krise durch Unterlassungen

Von Berthold Becher

SOZIALwirtschaft 3/2013, Seite 14-15

\section{Nachfolge}

Gesucht, gefunden!

Von Thomas Müller und Gabriele Moos

SOZIALwirtschaft 3/2013, Seite 16-18

\section{Fachkräfte-Befragung}

$\mathrm{Ob}$ das noch lange gutgeht?

Von Michael Knoll, Carsten Kuniß, Markus Burkhardt SOZIALwirtschaft 3/2013, Seite 19-20

\section{Finanzierungssysteme}

Schlangen, Studenten und ein Bier - ein Problemaufriss Von Bernd Halfar

SOZIALwirtschaft 4/2013, Seite 7-9

"Lesen, verstehen, ausprobieren!"

Interview mit Joachim Hagelskamp

SOZIALwirtschaft 4/2013, Seite 10

\section{Innovationen}

Sozial investieren

Von Berthold Becher

SOZIALwirtschaft 4/2013, Seite 11-15

Fördermittel

Stiftungen und Europäische Union als Zuschussgeber

Von Torsten Schmotz

SOZIALwirtschaft 4/2013, Seite 16-19

Rechnungswesen

Kosten kennen und belegen

Von Jürgen Holdenrieder und Nina Maier

SOZIALwirtschaft 4/2013, Seite 20-23

\section{Methoden}

Wirkungsforschung ist machbar

Von Michael Macsenaere

SOZIALwirtschaft 5/2013, Seite 7-10

\section{Social Return on Investment}

Gesellschaftliche Kosten und individuelle Wirkungen

Von Britta Wagner

SOZIALwirtschaft 5/2013, Seite 11-14

\section{Sozialbilanzen}

Auf der Suche nach den "richtigen « Indikatoren

Von Michael Weber

SOZIALwirtschaft 5/2013, Seite 15-17

Eingliederungshilfe

Ziele und Kennwerte evaluieren

Von Michael Scheer und Anton Bartling

SOZIALwirtschaft 5/2013, Seite 18-20 


\section{Organizational Burnout}

Ausgebrannte Organisationen

Von Gustav Greve

SOZIALwirtschaft 5/2013, Seite 21-23

Soziale Innovation

Zwischen Modebegriff und Zukunftstrend

Von Stefan Spieker

SOZIALwirtschaft 6/2013, Seite 7-10

\section{Positionen}

Innovationen in der Freien Wohlfahrtspflege

Von Gerhard Timm

SOZIALwirtschaft 6/2013, Seite 11-12

\section{Vernetzung}

Innovationen in traditionellen Strukturen

Von Stefan Zollondz

SOZIALwirtschaft 6/2013, Seite 13-15

\section{Design Thinking}

Wie man auf neue Gedanken kommt

Von Johannes Meyer

SOZIALwirtschaft 6/2013, Seite 16-18

\section{MAGAZIN}

\section{Arbeitsethos}

Fehler machen Spaß!

Von Simone Janson

SOZIALwirtschaft 1/2013, Seite 17-18

\section{Führung}

Ziele müssen »smart« sein

Von Matthias K. Hettl

SOZIALwirtschaft 1/2013, Seite 19-20

\section{Facility Management}

Herausforderung Klimatechnik

Von Thomas Heß

SOZIALwirtschaft 1/2013, Seite 21-22

\section{Sozialinformatik}

Open Source: Unentdeckte Potenziale

Von Peter Faiß

SOZIALwirtschaft 1/2013, Seite 23-25

\section{Bernd Halfar im Gespräch}

"Wir müssen Leistungen zukünftig anders erbringen«

Interview mit Renate Pfautsch

SOZIALwirtschaft 2/2013, Seite 21-23

\section{Krisenmanagement}

Die Guten tun sich schwer mit Kritik

Von Adrian Teetz

SOZIALwirtschaft 2/2013, Seite 24-26

\section{Bernd Halfar im Gespräch}

»Festzustellen ist eine zunehmend zukunftsfokussierte Ausrichtung «

Interview mit Gerhard Wiesheu

SOZIALwirtschaft 3/2013, Seite 21-22

Pflege

Aktionen allein helfen nicht

Von Mona Frommelt und Roland Schmidt

SOZIALwirtschaft 3/2013, Seite 23-27

\section{Bernd Halfar im Gespräch}

»Humor ist unverzichtbar"

Interview mit Dieter Czogalla

SOZIALwirtschaft 4/2013, Seite 24-25

\section{Kundenbindung}

Nutzer wollen gewonnen werden

Von Susanne Vaudt

SOZIALwirtschaft 4/2013, Seite 26-29

\section{Diversity}

Verschiedenheit managen

Von Andrea Tabatt-Hirschfeldt

SOZIALwirtschaft 5/2013, Seite 24-27

\section{Bernd Halfar im Gespräch}

»Die Sozialwirtschaft braucht die europäische Perspektive»

Interview mit Heinz Gerstlauer

SOZIALwirtschaft 6/2013, Seite 19-21

\section{Fachkräftemangel}

Abwarten ist keine Lösung

Von Matthias König, Hartmut Clausen und Christoph Schank

SOZIALwirtschaft 6/2013, Seite 22-25

\section{RUBRIKEN}

\section{Sozialpolitik}

Inklusion

Was uns hemmt

Von Stefan Blank

SOZIALwirtschaft 1/2013, Seite 26-27

\section{Inklusion}

Was passieren muss

Von Stefan Blank

SOZIALwirtschaft 2/2013, Seite 28-29

Tafeln

20 Jahre Tafeln: Keine institutionalisierte Dauerlösung

Von Stefan Selke

SOZIALwirtschaft 3/2013, Seite 28-29

\section{Inklusion}

Empowerment braucht Change-Management

Von Beate Blank

SOZIALwirtschaft 4/2013, Seite 30-31

\section{Wohlfahrtskonzepte}

Beziehungen statt Produkte

Von Brigitte Reiser

SOZIALwirtschaft 5/2013, Seite 28-29

Branchentarifvertrag

Ziel ist eine Stärkung der Branche

Von Bernd Schlüter

SOZIALwirtschaft 6/2013, Seite 26-27

\section{Personal}

\section{Führungskräfte-Nachwuchs}

Lernen im Tandem

Von Cora Schmidt

SOZIALwirtschaft 1/2013, Seite 28-29

Lohn

Ein Unternehmen braucht ein einheitliches und transparentes

Grundvergütungsmodell

Von Ralph Wißgott

SOZIALwirtschaft 2/2013, Seite 30-31

\section{Mitarbeitergespräch}

Mitarbeitergespräch: Wertvolles Instrument statt lästige

Pflicht

Von Dietgard Salein

SOZIALwirtschaft 3/2013, Seite 30-31

\section{Leitung}

Führen ohne Weisungsbefugnis

Von Johann Scholten

SOZIALwirtschaft 4/2013, Seite 32-33

\section{Low Performer}

Minderleitung führt zu Minderleistung

Von Friedhelm Wensing

SOZIALwirtschaft 5/2013, Seite 30-31

Betrieblicher Gesundheitförderung

Wohlbefinden mit Steuervorteil

Von Tobias Grambow

SOZIALwirtschaft 6/2013, Seite 28-29

\section{Finanzen}

\section{Bußgeld-Marketing}

Erfolgreich Geldauflagen einwerben 
Von Wilhelm Herrmann

SOZIALwirtschaft 1/2013, Seite 30-31

\section{Europäische Union}

Ein neuer Fonds gegen Armut und soziale Ausgrenzung Von Henning Braem

SOZIALwirtschaft 2/2013, Seite 32-33

\section{Fördermittel}

Für jedes Projekt gibt es Ansprechpartner

Von Beatrix Vogt-Wuchter

SOZIALwirtschaft 3/2013, Seite 32-33

\section{Zahlungsverkehr}

SEPA kommt

Von Bernd Bauer

SOZIALwirtschaft 4/2013, Seite 34-35

\section{Sponsoring}

Fundraising statt Spendenwerbung

Von Kai Dörfner

SOZIALwirtschaft 6/2013, Seite 30-31

\section{Recht}

\section{Gesetze}

Alles, was Recht ist

Von Bernd Bürger

SOZIALwirtschaft $1 / 2013$, Seite 32-33

\section{Holding}

Neue Aspekte für die Sozialwirtschaft (1)

Von Stefan Schick und Benjamin Liedy

SOZIALwirtschaft 2/2013, Seite 34-35

\section{Holding}

Neue Aspekte für die Sozialwirtschaft (2)

Von Stefan Schick und Benjamin Liedy

SOZIALwirtschaft 3/2013, Seite 34-35

Integrationsprojekte

Mehr Klarheit durch neue Bestimmungen

Von Andreas Seeger und Tilo Kurz

SOZIALwirtschaft 4/2013, Seite 36-37

\section{Betriebsrat}

Mitbestimmung in der Sozialwirtschaft

Von Tobias Grambow

SOZIALwirtschaft 5/2013, Seite 32-33

\section{Datenschutz}

Wir haben etwas zu verbergen!

Von Gerfried Riekewolt

SOZIALwirtschaft 6/2013, Seite 32-33

\section{Organisationsentwicklung}

\section{Interims-Management}

Führung auf Zeit

Von Edmund Fröhlich

SOZIALwirtschaft 1/2013, Seite 34-35

\section{Integrationsfirmen}

Integrationsfirmen als Lernfelder für Inklusion

Von Berthold Sommer

SOZIALwirtschaft 2/2013, Seite 36-37

Inklusion

Gewandelte Strukturen brauchen verändertes Denken Von Ludger Kolhoff

SOZIALwirtschaft 3/2013, Seite 36-37

\section{Sozialinformatik}

\section{Verwaltung}

Das papierlose Büro rechnet sich

Von Stephan Böck

SOZIALwirtschaft 5/2013, Seite 36-37

\section{Kompetenzen}

Was ein IT-Experte können muss

Von Wolfgang Boelmann und Helmut Kreidenweis

SOZIALwirtschaft 6/2013, Seite 36-37

\section{Marketing}

\section{Konzepte}

Mit Profil in den Wettbewerb

Von Thomas Hilse

SOZIALwirtschaft 1/2013, Seite 36-37

Leichte Sprache

Nutzer besser erreichen

Von Annette Flegel

SOZIALwirtschaft 5/2013, Seite 34-35

\section{Medien}

Wie man ein Video produziert

Von Olaf Olbricht

SOZIALwirtschaft 6/2013, Seite 34-35

\section{Literatur}

Jan Wulf-Schnabel: Reorganisation und Subjektivierungen von Sozialer Arbeit

Vorgestellt von Claus Melter

SOZIALwirtschaft 1/2013, Seite 38-39

Adrian Teetz: Krisenmanagement

Vongestellt von Harald Christa

SOZIALwirtschaft 2/2013, Seite 38-39

Herbert Bassarak, Sebastian Noll (Hg.): Personal im Sozialmanagement

Vorgestellt von Armin Müller

SOZIALwirtschaft 3/2013, Seite 38-40

Andreas Strunk (Hg.): Leitbildentwicklung und systemisches Controlling

Vorgestellt von Markus Bienecker

SOZIALwirtschaft 4/2013, Seite 38-40

Michael J. Sandel: Was man für Geld nicht kaufen kann

Vorgestellt von Harald Christa

SOZIALwirtschaft 5/2013, Seite 38-41

Sina Slottke: Social Entrepreneurship als multidimensionales Phänomen

Vorgestellt von Katrin Schneiders

SOZIALwirtschaft 6/2013, Seite 38-39

\section{Mein Buch}

Philip Kotler, Kevin Lane Keller, Friedhelm Bliemel: MarketingManagement. Strategien für wertschaffendes Handeln

Vorgestellt von Ingo Habenicht

SOZIALwirtschaft 1/2013, Seite 41

Leo Bormans (Hg.): Glück

Vorgestellt von André Peters

SOZIALwirtschaft 2/2013, Seite 41

Johannes Rüegg-Stürm: Das neue St. Galler Management-Modell

Vorgestellt von Dierk Starnitzke

SOZIALwirtschaft 3/2013, Seite 41

Christa Maurer: Mit Magellan und Captain Cook auf der Kommandobrücke. Was moderne Führungskräfte von erfolgreichen Kapitänen lernen können.

Vorgestellt von Karlheinz Petersen

SOZIALwirtschaft 4/2013, Seite 41

Abtprimas Notker Wolf, Schwester Enrica Rosana: Die Kunst, Menschen zu führen

Vorgestellt von Christian Reuter

SOZIALwirtschaft 6/2013, Seite 41

\section{AUTORINNEN UND AUTOREN}

Bartling, Anton

SOZIALwirtschaft 5/2013, Seite 18-20

Bauer, Bernd

SOZIALwirtschaft 3/2013, Seite 34-35

Becher, Berthold Dr.

SOZIALwirtschaft 3/2013, Seite 14-15 
SOZIALwirtschaft 4/2013, Seite 11-15

\section{Bienecker, Markus}

SOZIALwirtschaft 4/2013, Seite 38-40

Blank, Beate Dr.

SOZIALwirtschaft 4/2013, Seite 30-31

Blank, Stefan

SOZIALwirtschaft $1 / 2013$, Seite 26-27

SOZIALwirtschaft 2/2013, Seite 28-29

Böck, Stephan

SOZIALwirtschaft 5/2013, Seite 36-37

Boelmann, Wolfgang

SOZIALwirtschaft 6/2013, Seite 36-37

Braem, Henning

SOZIALwirtschaft 2/2013, Seite 32-33

Bürger, Bernd

SOZIALwirtschaft 1/2013, Seite 32-33

Burkhardt, Markus

SOZIALwirtschaft 3/2013, Seite 19-20

Christa, Harald Prof. Dr.

SOZIALwirtschaft 2/2013, Seite 38-39

SOZIALwirtschaft 5/2013, Seite 38-41

Clausen, Hartmut

SOZIALwirtschaft 6/2013, Seite 22-25

Czogalla, Dieter

SOZIALwirtschaft 4/2013, Seite 24-25

Dörfner, Kai

SOZIALwirtschaft 6/2013, Seite 30-31

Evans, Michaela

SOZIALwirtschaft 3/2013, Seite 7-9

Faiß, Peter

SOZIALwirtschaft 1/2013, Seite 23-25

Flegel, Annette

SOZIALwirtschaft 5/2013, Seite 34-35

Fröhlich, Edmund

SOZIALwirtschaft 1/2013, Seite 34-35

Frommelt, Mona

SOZIALwirtschaft 3/2013, Seite 23-27

Galchenko, Vlacheslav

SOZIALwirtschaft 3/2013, Seite 7-9

Gerstlauer, Heinz

SOZIALwirtschaft 6/2013, Seite 19-21

Grambow, Tobias

SOZIALwirtschaft 5/2013, Seite 32-33

SOZIALwirtschaft 6/2013, Seite 28-29

Greve, Gustav

SOZIALwirtschaft 5/2013, Seite 21-23

Gründel, Sascha

SOZIALwirtschaft 1/2013, Seite 10-12

Habenicht, Ingo Dr.

SOZIALwirtschaft 1/2013, Seite 41

Hagelskamp, Joachim

SOZIALwirtschaft 4/2013, Seite 10

Halfar, Bernd Prof. Dr.

SOZIALwirtschaft 2/2013, Seite 21-23

SOZIALwirtschaft $3 / 2013$, Seite 21-22

SOZIALwirtschaft 4/2013, Seite 7-9

SOZIALwirtschaft 4/2013, Seite 24-25

SOZIALwirtschaft 6/2013, Seite 19-21

Herrmann, Wilhelm

SOZIALwirtschaft 1/2013, Seite 30-31

Hess, Thomas

SOZIALwirtschaft 1/2013, Seite 21-22
Hettl, Matthias

SOZIALwirtschaft 1/2013, Seite 19-20

Hilbert, Josef Dr.

SOZIALwirtschaft 3/2013, Seite 7-9

Hilse, Thomas Dr.

SOZIALwirtschaft 1/2013, Seite 36-37

Hoffmann, Wolfgang

SOZIALwirtschaft 3/2013, Seite 10-13

Hofmann, Tina

SOZIALwirtschaft 2/2013, Seite 18-20

Holdenrieder, Jürgen Prof. Dr.

SOZIALwirtschaft 4/2013, Seite 20-23

Janson, Simone

SOZIALwirtschaft 1/2013, Seite 17-18

Kelter, Martin

SOZIALwirtschaft 1/2013, Seite 7-9

Knoll, Michael Dr.

SOZIALwirtschaft 3/2013, Seite 19-20

Kolhoff, Ludger Prof. Dr.

SOZIALwirtschaft 3/2013, Seite 36-37

König, Matthias Dr.

SOZIALwirtschaft 6/2013, Seite 22-25

Kreidenweis, Helmut Prof.

SOZIALwirtschaft 6/2013, Seite 36-37

Kuniß, Carsten

SOZIALwirtschaft 3/2013, Seite 19-20

Kurz, Tilo

SOZIALwirtschaft 4/2013, Seite 36-37

Liedke, Klaus-D.

SOZIALwirtschaft 2/2013, Seite 15-17

Liedy Benjamin Dr.

SOZIALwirtschaft $1 / 2013$, Seite 13-15

SOZIALwirtschaft 2/2013, Seite 34-35

SOZIALwirtschaft 3/2013, Seite 34-35

Macsenaere, Michael, Prof. Dr.

SOZIALwirtschaft 5/2013, Seite 7-10

Maier, Nina Dr.

SOZIALwirtschaft 4/2013, Seite 20-23

Melter, Claus Prof. Dr.

SOZIALwirtschaft 1/2013, Seite 38-39

Meyer, Johannes

SOZIALwirtschaft 6/2013, Seite 16-18

Moos, Gabriele Prof. Dr.

SOZIALwirtschaft 3/2013, Seite 16-18

Müller, Armin Dr.

SOZIALwirtschaft 3/2013, Seite 38-40

Müller, Thomas

SOZIALwirtschaft 3/2013, Seite 16-18

Olbricht, Olaf

SOZIALwirtschaft 6/2013, Seite 34-35

Peters, André

SOZIALwirtschaft 2/2013, Seite 41

Petersen, Karlheinz

SOZIALwirtschaft 3/2013, Seite 41

Pfautsch, Renate

SOZIALwirtschaft 2/2013, Seite 21-23

Raß, Günter

SOZIALwirtschaft 2/2013, Seite 13-14

Reiser, Brigitte Dr.

SOZIALwirtschaft 2/2013, Seite 10-12 SOZIALwirtschaft 5/2013, Seite 28-29

Reuter, Christian

SOZIALwirtschaft 6/2013, Seite 41
Riekewolt, Gerfried

SOZIALwirtschaft 6/2013, Seite 32-33

Salein, Dietgard

SOZIALwirtschaft 3/2013, Seite 30-31

Schank, Christoph Dr.

SOZIALwirtschaft 6/2013, Seite 22-25

Scheer, Michael

SOZIALwirtschaft 5/2013, Seite 18-20

Schick, Stefan Prof. Dr.

SOZIALwirtschaft 2/2013, Seite 34-35

SOZIALwirtschaft 3/2013, Seite 34-35

Schlüter, Bernd Prof. Dr.

SOZIALwirtschaft 6/2013, Seite 26-27

Schmidt, Cora

SOZIALwirtschaft 1/2013, Seite 28-29

Schmidt, Roland Prof. Dr.

SOZIALwirtschaft 3/2013, Seite 23-27

Schmotz, Torsten

SOZIALwirtschaft 4/2013, Seite 16-19

Schneiders, Katrin Prof. Dr.

SOZIALwirtschaft 6/2013, Seite 38-39

Scholten, Johann

SOZIALwirtschaft 4/2013, Seite 32-33

Seeger, Andreas

SOZIALwirtschaft 4/2013, Seite 36-37

Selke, Stefan Prof. Dr.

SOZIALwirtschaft 3/2013, Seite 28-29

Sommer, Berthold

SOZIALwirtschaft 2/2013, Seite 36-37

Spieker, Stefan

SOZIALwirtschaft 6/2013, Seite 7-10

Starnitzke, Dierk Prof. Dr.

SOZIALwirtschaft 3/2013, Seite 41

Strunk, Andreas Prof. Dr.

SOZIALwirtschaft 2/2013, Seite 7-9

Tabatt-Hirschfeldt, Andrea Prof. Dr.

SOZIALwirtschaft 5/2013, Seite 24-27

Teetz, Adrian

SOZIALwirtschaft 2/2013, Seite 24-26

Timm, Gerhard Dr.

SOZIALwirtschaft 6/2013, Seite 11-12

Vaudt, Susanne Prof. Dr.

SOZIALwirtschaft 4/2013, Seite 26-29

Vogt-Wuchter, Beatrix

SOZIALwirtschaft 3/2013, Seite 32-33

Wagner, Britta Dr.

SOZIALwirtschaft 5/2013, Seite 11-14

Walter, Jochen Dr.

SOZIALwirtschaft 2/2013, Seite 13-14

Weber, Michael Dr.

SOZIALwirtschaft 5/2013, Seite 15-17

Wensing, Friedhelm

SOZIALwirtschaft 5/2013, Seite 30-31

Wiesheu, Gerhard

SOZIALwirtschaft 3/2013, Seite 21-22

Winter, Sandra

SOZIALwirtschaft 1/2013, Seite 10-12

Wißgott, Ralph

SOZIALwirtschaft 2/2013, Seite 30-31

Zollondz, Stefan

SOZIALwirtschaft 6/2013, Seite 13-15 
vationsfähigkeit, Attraktivität für die richtigen Leute, zukünftige Verpflichtungen) einzustellen. (4)

Können wir heute beispielsweise im Bereich der Pflegeversicherung Leistungen ausbauen, wenn schon jetzt absehbar ist, dass das heutige Leistungsniveau in der heutigen Form nicht auf Dauer solidarisch finanziert werden kann? Haben Führungskräfte nicht bereits auch heute Verantwortung für die Konsequenzen, die mit der absehbaren Bugwelle des demografischen Wandels bisherige Versorgungsstrukturen und Einrichtungen substanziell gefährden und neue Lösungen erfordern werden.

Gerade in dieser Dimension der Verantwortung wird auch die zeitliche Komponente verantwortlichen Handelns deutlich. Müssen nicht unpopuläre Entscheidungen durch Führungskräfte getroffen und durchgehalten werden, wenn diese positive und notwendige Beiträge für die Entwicklung des Unternehmens oder die Gesellschaft leisten? Sind nicht historisch falsche Entscheidungen oder unzureichenden Annahmen im Management ursächlich, wenn eine soziale Einrichtung heute nicht mehr lebensfähig ist? Und sieht man nicht erst mit einem zeitlichen Versatz, ob Führungskräfte heute wirklich verantwortlich handeln?

Chancen und Risiken sozialer Unternehmen sind somit kontinuierlich durch Führungskräfte zu managen. Historische Aspekte sind genauso zu bedenken und heutige Situationen zu analysieren wie zukünftige Konsequenzen zu berücksichtigen. Die Schaffung einer ausreichenden Klarheit und Transparenz des Handelns von Führungskräften und die Dokumentation von Entscheidungsgrundlagen wird notwendige Voraussetzung sein, um diese Beurteilung auch über Jahre hinweg vornehmen zu können.

\section{Vierte Dimension verantwortungsvollen Managements: Wachstum der Mitarbeitenden}

»Ethik im Management von Personen heißt, es Menschen zu ermöglichen, Leistungen dort zu erbringen, wo sie ihre Stärken haben und dadurch erfolgreich zu sein. «(5)

Führungskräfte stehen in der Verantwortung, Mitarbeitende mit den richtigen Aufgaben zu betrauen. Aufgaben, die auch dadurch, dass es große und verantwortungsvolle Aufgaben sind, das Wachstum von Mitarbeitenden fördert. Diese Chance des fachlichen und persönlichen Wachstums von Menschen stellt die notwendige Grundlage für die Weiterentwicklung und Verbesserung der Leistungen für die Menschen und sogar der Entwicklung der gesamten Organisation dar.

Ein solches Potenzial darf durch eine wachstumshemmende Führungskultur, die mit Angst, Druck, fehlender Kontinuität von Entscheidungen, unzureichender Verlässlichkeit, mangelndem Vertrauen und übermäßiger Kontrolle arbeitet, nicht aufs Spiel gesetzt werden. Vorbildliche Vorgesetzte vertrauen daher auf Menschen und Beziehungen, nicht auf Regeln und Strukturen. Sie sehen ihre Beschäftigten als eigenverantwortliche Profis, die Aktivitäten koordinieren, integrieren und sich auch weiterentwickeln können. (6)

\section{Fünfte Dimension verantwortungsvollen Managements: Eigene Leistungsfähigkeit und Gesundheit fördern}

Traditionell haben sich Erziehung, Pädagogik und auch die Managementausbildung ausschließlich auf die Anforderung der Übernahme einer sozialen Verantwortung konzentriert. Die persönliche Verantwortung für die eigene physische, psychische und mentale Gesundheit ist erst in neueren Ausbildungs-Curricula für Führungskräfte zu finden. Dabei bildet diese persönliche Verantwortung für die eigene Leistungsfähigkeit und Gesundheit die Grundlage für die wirksame und nachhaltige Übernahme sozialer Verantwortung. (7)

Eine Führungskraft kann zwar Verantwortung für die Menschen, die Umwelt, das Unternehmen und die Mitarbeiterschaft im Übermaß erfüllen, doch wird dies nicht auf Dauer gelingen, wenn die eigene Gesundheit früher sträflich vernachlässigt wird und dies aus dem Blick gerät. Früher oder später rächt sich eine solche Ignoranz und markiert zuweilen plötzlich und unerwartet die Grenzen der Leistungsfähigkeit.

Hierbei ist - wie immer bei Fragen der Verantwortlichkeit - nicht nur das Verhalten der Führungskraft zu betrachten, was der eigenen Gesundheit zuträglich ist, sondern auch das was jemand an notwendigen Maßnahmen zur Gesundheitsförderung unterlässt. »Verantwortlich ist mithin jeder nicht nur für das, was er tut, sondern auch für das was er unterlässt.« (8)

Verantwortung in eigener Sache zu übernehmen ist deshalb ein weiteres Muss für Führungskräfte und (hoffentlich) ein probates Mittel drohenden Burn-out-Prozessen entgegenzuwirken.

\section{Fazit}

»Im Mittelpunkt des Managements steht das Element der Verantwortung, als wesentlicher Teil einer guten Unternehmenskultur und Unternehmensethik. «(9)

Verantwortung, die man von Führungskräften zwar erwarten kann, bei der jedoch immer die persönliche Entscheidung der Führungskraft selbst entscheidend ist, nämlich ob sie sich dieser komplexen und multipolaren Herausforderung stellt oder sich immer wieder auch der Verantwortung entzieht. Das hier vorgeschlagene Konzept bietet für Führungskräfte eine Orientierungshilfe sich dieser Herausforderung professionell zu stellen. Es bietet die Chance voreilige oder einseitige Beurteilungen $\mathrm{zu}$ vermeiden.

Die Überlegungen könnten auch einen Beitrag für die Verankerung einer mehrdimensionalen Verantwortungsund Führungskultur - nicht nur in Sozialunternehmen - sein und somit auch ein wertvolles Instrument für Aufsichtsgremien werden.

\section{Anmerkungen}

(1) Drucker, P. (2009): Management, Bd. 1, S. 168.

(2) Vgl.: Sellner, C. (2004): Nachhaltigkeit (k)ein Thema für Sozialunternehmen, S. 54.

(3) Drucker, P. (2009): Management, Bd. 2, S. 358.

(4) Vgl. Malik, F. (2008): Unternehmenspolitik, S. 176.

(5) Malik, F. (2008): Unternehmenspolitik, S. 236.

(6) Vgl.: Kanter, R. M.: Anders Wirtschaften, in Harvard Business Manager, Febuar 2012, S. 36.

(7) Vgl.: Juul, J.: Dein kompetentes Kind, 2009, S. 140.

(8) Sprenger, R. K. (1999): Prinzip Selbstverantwortung, S. 47.

(9) Vgl.: Malik, F. 2008: Unternehmenspolitik, S. 236. 